\title{
Proactive interference in monkeys: Delay and intersample interval effects are noncomparable
}

\author{
DOUGLAS L. MEDIN \\ University of Illinois, Champaign, Illinois 61820
}

\begin{abstract}
Two prominent theories of proactive interference in animal memory predict that the effects of varying the interval between the interfering and to-be-remembered stimulus in a delayedmatching-to-sample paradigm ought to be comparable to the effects of manipulating the retention interval. To assess this prediction, monkeys were tested in a situation in which a sample was presented, followed by a variable intersample interval, whereupon a second sample was presented. After a delay interval, a choice test was given between the two stimuli that had served as samples. The correct choice was always the most recently presented sample stimulus, and the initial sample of a sequence provided a potential source of proactive interference. In two experiments, delay interval altered performance, whereas interstimulus interval had little or no effect. In a third experiment, using a small set of sample stimuli, intertrial interval altered proactive interference, but again interstimulus interval had no effect. One way of accounting for these data is in terms of distinct short- and long-term memory processes.
\end{abstract}

As interest in short-term memory in animals continues, the number of stable empirical relationships uncovered steadily mounts. There is evidence, for example, that interference treatments alter memory for stimuli, responses, and reinforcers in a similar way (Maki, Moe, \& Bierley, 1977) and produce the same pattern of results regardless of whether the subject population consists of pigeons, monkeys, or dolphins (D'Amato,1973; Herman, 1975; Roberts \& Grant, 1976; Wilson, 1974; Worsham, 1975; Zentall \& Hogan, 1974). Not surprisingly, there has been a concomitant interest in theories of animal memory, especially as they apply to interference phenomena.

In discussing contrasting theories it will be useful to focus on a concrete experimental situation. Consider a delayed-matching-to-sample (DMTS) paradigm with two kinds of trials: (1) control trials, in which sample stimulus $B$ is presented and, after a delay (retention) interval, a choice between stimulus $A$ and stimulus B is given, with stimulus B rewarded; or (2) proactive interference trials, in which stimulus $A$ is presented, then stimulus $B$ is presented, and, after a delay interval, $\mathbf{A}$ and $\mathbf{B}$ are given on the choice test with stimulus B rewarded. Over an experimental session, $A$ and $B$ are rewarded equally often so that the correct solution is to choose the stimulus that has most recently served as a sample. Typically, performance on control trials surpasses that on inter-

This research was supported by U.S. Public Health Service Grants MH 25134 and MH 32489. Thomas J. Reynolds provided helpful comments during the preparation of this paper. Requests for reprints should be sent to Douglas Medin, Psychology Department, University of Illinois, Champaign, Illinois 61820. ference trials. Any memory theory must be able to address this basic finding.

Although this paradigm seems to provide a natural tool for examining relationships between short-and long-term memory, the two most prominent theories of DMTS (D'Amato, 1973; Roberts \& Grant, 1976) do not assume that performance reflects separate contributions from short- and long-term memory. Roberts and Grant, for example, proposed that longterm memory does not enter into pigeon DMTS performance. Instead, they assume that performance is controlled by the strengths of memory traces in short-term memory. Similarly, D'Amato's suggestion that DMTS performance involves a relative recency judgment or temporal discrimination does not rest on the assumption of separate memory stores. Either theory could account for proactive interference effects (see Reynolds \& Medin, 1979, for a more detailed discussion of these theories and relevant data).

One means of evaluating the merits of adding the distinction between short-term processing and longterm memory in DMTS performance is by attempting to find circumstances in which the absence of such a distinction leads the theories to make erroneous predictions. The present experiments examined the relative effects of delay and intersample presentation intervals on DMTS in monkeys. On a typical trial, stimulus $\mathbf{A}$ is presented and rewarded; following a variable intersample interval, stimulus $\mathbf{B}$ is presented and rewarded; and after a variable delay interval, both stimuli are presented for the choice test, with stimulus B being correct and rewarded.

The major concern in these experiments is the relationship between intersample interval effects and 
delay effects. According to both strength and temporal discrimination theories, performance should decrease with delay and increase with ISI (intersample interval) in a straightforward way. According to temporal discrimination theory, as ISI increases, it should be easier to determine the temporal order of $\mathrm{A}$ and $\mathrm{B}$, and strength theory predicts a facilitation because the longer the ISI, the more chance for the strength of stimulus A to decay. Moreover, these theories expect a rather precise relation between ISI and delay effects. For example, the strength of B at a 36-sec delay should be approximately the same as the strength of A after an 18-sec ISI and an 18-sec delay interval, because the Roberts and Grant theory posits that the strengths of $A$ and $B$ are independent.

Consider now what might be expected on the basis of analyzing stimulus processing in terms of two memory stores. Test performance would be assumed to be based either on the more stable long-term information, if it can be accessed, or on short-term memory, if the appropriate information still resides there. The Wagner, Rudy, and Whitlow (1973) theory of rehearsal in animal conditioning, as well as Spear's $(1973,1976)$ analysis of stimulus processing in relation to reinstatement or retrieval cues, is consistent with this general framework. Suppose that poststimulus processing (rehearsal) tends to occur following sample presentations and that, on interference trials, the presentation of the B stimulus disrupts rehearsal of the A stimulus in favor of rehearsal of the B stimulus. In that event, test performance will reflect contributions from both short- and long-term memory for stimulus B, but only contributions from longterm memory for stimulus $A$. On this basis, one would expect delay effects arising from the loss of information concerning stimulus $\mathbf{B}$ from short-term memory, but one would not necessarily expect comparable ISI effects. If rehearsal of stimulus A increased its strength in long-term memory, then ISIs should tend to lower performance because of the extra time for strengthening A. A potential compensatory factor is that longer ISIs involve longer periods of time between the presentation of stimulus $\mathrm{A}$ and the choice test, and forgetting from longterm memory would tend to diminish rehearsal effects. ${ }^{1}$ Thus, this alternative account does not expect that ISI and delay effects will be commensurate; in particular, delay effects will be larger and more systematic than ISI effects.

\section{EXPERIMENT 1}

The first experiment employed the three main types of DMTS trials shown in Figure 1. On all choice tests, the stimulus which had most recently served as the sample was correct and rewarded. The sample stimulus that was to be correct was presented twice in immediate succession when it appeared as the sample. This procedure was designed to improve
Single Somple Control

Double Stimulus Control

Double Stimulus Interference

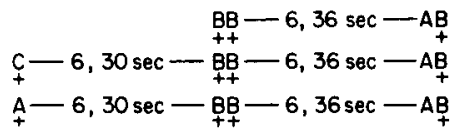

Figure 1. Design of Experiment 1. ISI refers to interstimulus interval. A different pair of objects appeared on each trial.

performance on interference trials and increase the likelihood of detecting ISI effects. Double sample control trials were included to evaluate effects of a prior sample that are not specific to its appearing on a choice test. New stimuli were used on every trial.

\section{Method}

\section{Subjects}

The subjects were 5- to 6-year-old jungle-born pig-tailed monkeys (Macaca nemestrina) and two 3- to 4-year-old jungle-born rhesus monkeys (Macaca mulatta). All animals had 2-3 years experience on various types of discrimination problems, including DMTS problems in which the correct object on a choice test was always the object that had served as the sample and been rewarded most recently.

\section{Apparatus}

A Wisconsin General Test Apparatus (WGTA) was employed for testing. The three foodwells of the gray formboard were spaced $15 \mathrm{~cm}$ apart, center-to-center. The 480 stimulus objects used in the experiment were selected from a pool of common-use and manufactured objects.

\section{Procedure}

Pretraining. Each day for 20 days of preliminary training, the animals were given 24 trials consisting of 6 control and 18 interference trials. On control trials, the following sequence of events occurred: (1) A single stimulus (the sample) was presented over the central foodwell; (2) the monkey displaced the object and received a raisin reward; (3) a delay interval of 6,18 , or $36 \mathrm{sec}$ ensued; and (4) the sample stimulus and a new stimulus appeared over the two side foodwells and a choice of the stimulus that had served as the sample was correct and rewarded with a raisin. For interference trials: (1) a single stimulus appeared over the central foodwell; (2) the monkey displaced the object and received a raisin reward; (3) an ISI interval of 6,18 , or $36 \mathrm{sec}$ ensued; (4) a second sample stimulus was presented over the central foodwell; (5) the monkey displaced the object for a raisin reward; (6) a delay interval of 6 , 18 , or 36 sec ensued; and (7) the two stimuli appeared over the side foodwells and the correct, rewarded stimulus was the object that had most recently served as the sample. A noncorrection procedure was used and the intertrial interval (ITI) was always $15 \mathrm{sec}$. The position of the rewarded object was randomized but balanced over each day. A new pair of objects was introduced on every trial.

Testing. Testing used stimuli drawn from the same pool of 480 common-use and manufactured objects. After all the objects were used, they were randomly mixed and new samples drawn. DMTS testing was given using the three problem formats shown in Figure 1. On all three types of trials, the stimulus that was to be correct on the choice test was presented twice over the center foodwell, with the interval between the two presentations being $6 \mathrm{sec}$, close to the minimum time for operating the WGTA. For single-sample control trials, the incorrect stimulus was introduced at the time of test. On double-stimulus control trials, the two presentations of the correct sample stimulus were preceded, by 6 or $30 \mathrm{sec}$, by a different sample, one that did not appear on the test. Interference trials differed from double-sample control trials in that the initial sample was the incorrect object on the choice test. The combination of ISI and delay intervals produced $10 \mathrm{kinds}$ of 
trials, each was presented exactly twice to each animal each day. On choice tests, the stimuli always appeared over the side foodwells and the position of the correct stimulus was randomized but balanced within a day. A new set of objects was used on every trial and the ITI was a constant $15 \mathrm{sec}$. Testing continued for 30 days.

\section{Results}

\section{Pretraining}

Performance during pretraining was worse on interference trials than on control trials and dropped as retention interval increased, but did not vary as a function of intersample interval. Control trial performance dropped from $83 \%$ correct at a 6-sec delay interval to $67 \%$ correct at a $36-\mathrm{sec}$ delay interval. Interference trial performance dropped from $71 \%$ correct to $62 \%$ correct over the same delay intervals. Animals averaged $67 \%$ correct responses for each of the ISIs. The overall low level of performance makes interpretation of these results somewhat difficult, but, in any case, delay and ISI effects were not comparable.

\section{Testing}

The main results are shown in Table 1 . The singlestimulus control condition produced the best performance, the double-stimulus control trials produced slightly poorer performance, and the interference trials were associated with the worst performance. There was also some tendency for long ISIs to produce better performance than short ISIs. Finally, the difference between control and interference trials appeared to increase with delay.

An analysis of variance indicated that the effects of Conditions $[\mathrm{F}(4,24)=11.34$, MSe $=3.17, \mathrm{p}<.01]$ and Delay $[\mathrm{F}(1,6)=12.21, \mathrm{MSe}=12.9, \mathrm{p}<.09]$ were significant, but neither the main effects of 10-day blocks of practice $(F<1)$ nor any of the interactions with these main variables were statistically reliable. Individual comparisons of the overall conditions effect, using Duncan's multiple range test and the .05 confidence level, indicated that the single-stimulus control condition produced better performance than the two interference conditions and that the two doublestimulus control conditions yielded better performance than the interference condition involving the short interpresentation interval.

Table 1

Proportion Correct as a Function of Trial Type and Delay Interval in Experiment 1

\begin{tabular}{lll}
\hline \multirow{2}{*}{$\begin{array}{c}\text { Trial } \\
\text { Type }\end{array}$} & \multicolumn{2}{c}{ Delay Interval (in Seconds) } \\
\cline { 2 - 3 } & 6 & 36 \\
\hline BB & .97 & .91 \\
C-6-BB & .94 & .84 \\
C-30-BB & .92 & .87 \\
A-6-BB & .88 & .72 \\
A-30-BB & .91 & .77 \\
\hline
\end{tabular}

Since there is some question of which control condition is the more appropriate, a second analysis was done dropping the single-stimulus control condition. For this ANOVA, the main effects of Control vs. Interference $[F(1,6)=19.85, \mathrm{MSe}=9.92, \mathrm{p}<.01]$ and Delay $[\mathrm{F}(1,6)=13.32$, MSe $=33.04, \mathrm{p}<.05]$ were significant, while the main effect of Intersample Interval fell short of significance $[F(1,6)=3.58$, $\mathrm{MSe}=3.12, \mathrm{p}>.10 \mathrm{]}$. None of the interactions involving these main effects approached significance.

There were considerable individual differences in the monkeys' performance. Two animals averaged $33 \%$ errors, whereas the other five averaged fewer than $15 \%$ errors. If the above ANOVA is applied to the performance of the five subjects with the best performance, then all three main effects (including ISI) are reliable and the Control vs. Interference by Delay Interval interaction approaches significance $[F(1,4)=6.35$, $\mathrm{MSe}=8.34, .05<\mathrm{p}<.10]$. The theoretically important interaction of Control vs. Interference with Intersample Interval, however, was far from significant $(F \approx 1)$.

\section{Discussion}

The mere presentation of two different sample stimuli was not sufficient to produce pronounced interference effects. The difference between single- and double-stimulus control trials was small and not statistically significant. Zentall and Hogan (1977) noted a similar small difference in a study using pigeons. All of the models under consideration are compatible with this main result. Double-stimulus control trials should alter performance only to the extent that the choice stimuli are similar to the extra sample.

Intersample interval was not a major factor in proactive interference. While the monkeys with excellent overall performance tended to make more errors on trials involving short ISIs, this held for both control and interference trials. Temporal discrimination and trace strength competition theories predict that performance should be better on interference trials involving a long rather than a short ISI. In no case, however, did the ISI by Control vs. Interference interaction approach statistical significance, and ISI effects predicted on the basis of delay effects did not materialize.

\section{EXPERIMENT 2}

In the DMTS situations employed by Roberts and Grant with pigeons and D'Amato with monkeys, responses to the sample presentation are not associated with rewards. In contrast, the present studies and other DMTS experiments using a WGTA typically reward responses to sample stimuli (e.g., Mason \& Wilson, 1974; Mishkin \& Delacour, 1975). A number of basic interference effects appear in both 
of these situations (see Reynolds \& Medin, 1979), but rewarding all samples makes the basis of performance somewhat ambiguous. With rewarded samples, performance may be based on the association of the sample stimulus with reward or it might simply be based on a learned tendency to choose the more familiar stimulus. In this paradigm, presentation frequency and reward frequency are perfectly correlated. Experiment 2 examined ISI effects in a situation in which performance must be based on reward information rather than familiarity. A sample presentation was either rewarded or nonrewarded, and the stimulus that served as the sample was correct when it was most recently rewarded and incorrect when it was most recently nonrewarded.

Although it is possible to extend temporal discrimination theory to a situation in which reward information controls performance, Experiment 2 seems to apply more directly to strength theories. Performance during the preliminary training in Experiment 2 bears on the question of whether responses in the first experiment were based on familiarity or reward value. Finally, Experiment 2 used the same stimulus (but differing outcomes) as both the correct sample and the interfering sample so that a direct comparison of proactive interference and repetition effects could be made. On control trials, the sample stimulus was presented twice with each sample presentation either rewarded or nonrewarded. Interference conditions arose when the reward status of the second sample presentation differed from that of the first. The correct solution always depended on the most recent reward status; if the sample was most recently rewarded, then it was correct on the choice test, while if the sample most recently was nonrewarded, then it was incorrect on the choice test.

\section{Method}

\section{Subjects and Apparatus}

The monkeys, test apparatus, and stimuli from the first experiment were used. One pigtail monkey completed only preliminary training for reasons unrelated to the experimental variables.

\section{Procedure}

Preliminary training. The basic trial sequence was as follows: (1) A sample was presented over the center foodwell; (2) displacing the object either was or was not associated with a raisin reward; (3) a choice between the sample and a new stimulus was given after a minimal (6-sec) delay interval; and (4) if the sample had been rewarded, it was correct and rewarded, but if the sample had not been rewarded, it was incorrect and responses to the alternative stimulus were rewarded. New objects were used on each trial, 24 trials a day for 20 days. The intertrial interval was approximately $8 \mathrm{sec}$, and reward conditions and stimulus positions were randomized but balanced within a day.

Critical testing. Three trial types were presented. On single-sample control trials, a single object was presented as the sample, responses to the sample were either rewarded or nonrewarded, and, after a delay of 6 or $36 \mathrm{sec}$, a choice test was given between the sample and a new stimulus. On repeated-sample control trials, the sample appeared twice, separated by a 6-, 12-, or 30-sec interval, and both responses to the sample were either rewarded or nonrewarded; after the delay interval, a choice test was given between the sample and a new stimulus. Repeated-sample interference trials were the same as repeated-sample controls, except that the reward conditions on the first and second presentation of the sample were different. For all three trials, the correct solution was to choose the stimulus that had been the sample if it had been most recently rewarded and to choose the new alternative if the sample was most recently associated with nonreward. The various combinations of trial types and interpresentation and delay intervals produced 28 different trial conditions, each of which was presented once each day in a random order. The ITI was constant at $15 \mathrm{sec}$, and position of the correct object on choice tests was random, but balanced, within a day. Each animal received 50 days of testing.

\section{Results}

\section{Pretraining}

The pattern of results in preliminary training strongly suggests that the monkeys' DMTS performance had been controlled by familiarity rather than reward associated with the sample stimulus. On the first day of training, the monkeys averaged $66 \%$ errors when the sample was not rewarded but only $14 \%$ errors when the sample was rewarded. Since learningset-experienced monkeys perform better on nonrewarded trials (for a review see Medin, 1972), this pattern is almost surely not the result of fundamental differences in the effectiveness of reward and nonreward. The monkeys were simply choosing the familiar stimulus on the choice tests.

Performance with nonrewarded samples rapidly improved over days. On the final day of training, the monkeys averaged $17 \%$ errors on rewarded trials and $18 \%$ errors on nonrewarded trials. By the end of training, performance had come to be based on whether or not the stimulus had been rewarded rather than stimulus familiarity. An analysis of variance indicated that the main effects of 5-day blocks of practice $[F(3,18)=12.79$, MSe $=13.79, p<.01]$ and reward vs. nonreward $[\mathrm{F}(1,6)=46.12$, MSe $=80.86$, $\mathrm{p}<.01]$ as well as their interaction $[\mathrm{F}(3,18)=9.67$, MSe $=29.97, p<.01]$ were significant.

\section{Critical Testing}

The main results are shown in Table 2. Significant interference effects are indicated by the overall difference between repeated-sample control trials and repeated-sample interference trials. Single-sample control trials produced intermediate performance. For repeated-sample control trials, performance did not seem to vary with ISI, and trials on which the sample was correct produced better performance than trials on which it had been incorrect. On interference trials, there was a small but consistent nonmonotone ISI effect, with performance being worst at intermediate intervals.

An analysis of variance on the repeated-sample trials using 25-day Practice Blocks, Control vs. Interference, Delay, Reward Condition, and Intersample Interval as factors indicated that the main effects of 
Table 2

Proportion Correct on Simple-Sample and Repeated-Sample Trials as a Function of Reward Status, Intersample Interval (ISI), and Delay Interval (DI)

\begin{tabular}{|c|c|c|c|c|c|c|}
\hline \multirow{3}{*}{$\begin{array}{l}\text { Trial } \\
\text { Type }\end{array}$} & \multicolumn{6}{|c|}{ ISI (in Seconds) } \\
\hline & \multicolumn{3}{|c|}{ 6-Sec DI } & \multicolumn{3}{|c|}{ 36-Sec DI } \\
\hline & 6 & 12 & 30 & 6 & 12 & 30 \\
\hline & \multicolumn{6}{|c|}{ Repeated-Sample Control } \\
\hline \multirow[t]{2}{*}{ Both Samples - } & $\begin{array}{l}.93 \\
.84\end{array}$ & $\begin{array}{l}.92 \\
.82\end{array}$ & $\begin{array}{l}.94 \\
.84\end{array}$ & $\begin{array}{l}.85 \\
.77\end{array}$ & $\begin{array}{l}.86 \\
.78\end{array}$ & $\begin{array}{l}.84 \\
.76\end{array}$ \\
\hline & \multicolumn{6}{|c|}{ Repeated-Sample Interference } \\
\hline \multirow[t]{2}{*}{ Second Sample - } & $\begin{array}{l}.74 \\
.74\end{array}$ & $\begin{array}{l}.70 \\
.67\end{array}$ & $\begin{array}{l}.74 \\
.72\end{array}$ & $\begin{array}{l}.64 \\
.70\end{array}$ & $\begin{array}{l}.58 \\
.63\end{array}$ & $\begin{array}{l}.59 \\
.70\end{array}$ \\
\hline & \multicolumn{6}{|c|}{ Single-Sample Control } \\
\hline $\begin{array}{l}\text { Sample + } \\
\text { Sample - }\end{array}$ & & $\begin{array}{l}.88 \\
.81\end{array}$ & & & $\begin{array}{l}.85 \\
.68\end{array}$ & \\
\hline
\end{tabular}

Note-ISIs are not applicable to the single-sample control condition. Each point is based on 300 observations.

Control vs. Interference $[\mathrm{F}(1,5)=157.34$, MSe $=$ $21.07, \mathrm{p}<.01]$, Delay $[\mathrm{F}(1,5)=10.33, \mathrm{MSe}=47.21$, $\mathrm{p}<.05]$, and Reward Condition $[\mathrm{F}(1,5)=23.41$, $\mathrm{MSe}=10.37, \mathrm{p}<.01]$ were significant. No other main effect or interaction proved to be reliable.

To further explore possible nonmonotone ISI effects, a trend analysis was conducted using the factors of Control vs. Interference and Intersample Interval. The quadratic component of the ISI effect proved to be significant $[\mathrm{F}(1,5)=11.21, \mathrm{MSe}=12.4$, $\mathrm{p}<.05$ ], but the quadratic component of the Intersample Interval by Control vs. Interference interaction fell short of statistical reliability $[F(1,5)=$ $4.15, \mathrm{MSe}=37.6, .05<\mathrm{p}<.10$ ]. Overall performance was worst for the intermediate ISIs.

\section{Discussion}

Experiment 2 produced a number of interesting results. First, performance during preliminary training initially was based on familiarity rather than reward value. By implication, performance in the first experiment was also based on familiarity. Perhaps this tendency to respond on the basis of familiarity underlies the general pattern of comparability between studies rewarding all sample presentations and studies using rewards only on choice tests.

A second major result was that reliable delay effects were again observed in the absence of improved performance on the longer ISIs. The data gave some indication that ISI effects were nonmonotonic. Performance at the intermediate ISI on interference trials was lower than performance at either the short or the long ISI. This result could provide a stringent test of alternative memory models. The effects are quite small, however, and the seriousness with which they must be taken depends on their future replicability.
Finally, there was no effect of ISI on repeatedsample control trials. Other experiments with monkeys have suggested that whether or not increasing ISI helps or hurts performance depends on whether a large or a small set of stimuli are employed (Herzog, Grant, \& Roberts, 1977; Medin, 1974). In fact, using some of the same monkeys employed in Experiment 2, Medin found that increasing ISI impaired performance when a small set of alternative objects was used and helped performance when a large set of objects was used (contrary to the present study). The present experiment differed from Medin's (1974) earlier experiment along a number of variables, such as number of sample presentations, length of delay intervals, and whether or not the sample presentations were invariably rewarded. One idea suggested by Herzog et al. (1977) is that the advantage of spaced presentations derives from differential encoding on the two appearances of the sample. One may speculate that, after several additional years of experience, the monkeys become less variable in their encoding of stimuli so that variability in encoding did not increase with ISI. A simple test of this idea would be to employ the procedures of Experiment 2 with a naive group of monkeys. Another interesting possibility is that whether or not spacing repetitions facilitates performance depends on which attribute underlies performance. It seems likely that familiarity was the basis of performance in Medin's (1974) experiment, while the present experiment required that reward information be the basis of performance. While these and similar speculations are unwarranted, at least they are testable and, obviously, more work is needed to clarify the various patterns of repetition effects.

\section{EXPERIMENT 3}

Although the first two experiments are reasonably clear, they are not especially compatible with other experiments varying ISI. Studies with pigeons reveal a somewhat uncertain picture concerning ISI effects. Grant and Roberts (1973) found very little difference between ISIs of 0 and $10 \mathrm{sec}$ when a FR 1 schedule was in effect on the sample stimulus, but a 0 -sec interval led to worse performance under a FR 30 schedule. In an experiment in which presentation time was experimenter-controlled, Roberts and Grant (1974) found no difference between ISIs of $0 \mathrm{vs.} 1 \mathrm{sec}$.

Jarvik, Goldfarb, and Carley (1969), using rhesus monkeys as subjects, found that delayed matching performance decreased with delay and improved as ISI lengthened, a result that is entirely consistent with strength and temporal discrimination theories. Jarvik et al. held constant the interval between the response on a test trial and the beginning of the next trial (that is, the intertrial interval was constant). Under this procedure, the intertest interval varies with ISI. If performance improves as the intertest interval 
increases, then the ISI effects found by Jarvik et al. may reflect contributions arising from the intertest interval. Another possibility is that ISI effects are observed only when a small set of sample stimuli is employed. None of the theories we have been considering implies this constraint, but this possibility bears examination. Experiment 3 addressed both of these possibilities.

Is there any theoretical basis for expecting the intertest interval to be an important factor in performance? A number of investigators (e.g., Medin, Reynolds, \& Parkinson, 1980; Spear, 1976; Zentall, 1970) have emphasized the importance of reinstatement and retrieval cues in memory processing. In a DMTS paradigm, the sample stimulus appears alone and in a different location (in the present experiment, over the center foodwell) compared with the choice test setting (in which the objects cover the outside foodwells). In other words, a considerable change in context separates sample and test presentations. Consecutive test trials, by comparison, may involve smaller changes in context. When only two alternative stimuli are used, the only change between consecutive test trials is that the position of the two choice stimuli may have been switched. Consequently, the preceding test trial may exert a substantial influence on current test trial performance. Increasing the interval between the preceding and the current test trial should facilitate performance, since the availability of information from the preceding test trial should decrease with time. In Experiments 1 and 2, the interval between consecutive tests was not controlled, but since those experiments used different stimuli on each trial, our analysis implies that there should be little interference between trials. The test stimuli on the current trial would not be good retrieval cues for the test stimuli on the preceding trial. Experiment 3 used a small set of sample stimuli, but the various experimental conditions allowed a separate estimate of ISI and intertest interval effects.

\section{Method}

\section{Subjects and Apparatus \\ The four pigtailed monkeys that completed Experiment 2 and one DMTS-experienced cynomolgus monkey (Macaca fascicularis) were used. Approximately 1 year intervened between Experiment 2 and Experiment 3, during which time the monkeys were moved to another laboratory and run in other DMTS experiments. In almost all these experiments, the most recently presented sample stimulus was correct, but the monkeys also had the experience in which the reward status of the sample determined which object was correct. Testing was conducted in a WGTA, with the only change being that the food tray was painted black rather than gray.}

\section{Procedure}

The experimental conditions outlined in Figure 2 were used in testing. The two conditions in the top part of the figure provide a direct test of intertest interval effects. Following an intertrial interval (ITI) of either 6 or $30 \mathrm{sec}$, a sample was presented and

\begin{tabular}{|c|c|c|c|}
\hline ITI & \multicolumn{2}{|c|}{ DELAY } & IEST \\
\hline $6 \mathrm{sec} \frac{B}{+}$ & \multicolumn{2}{|c|}{$6,30 \mathrm{sec}$} & A $\underset{+}{B}$ \\
\hline $30 \mathrm{sec} \stackrel{\mathrm{B}}{+}$ & \multicolumn{2}{|c|}{$6,30 \mathrm{sec}$} & $A \underset{+}{B}$ \\
\hline ITI & ISI & DELAY & TEST \\
\hline $30 \sec A$ & $6 \mathrm{sec} B$ & $30 \mathrm{sec}$ & A $\underset{T}{ }$ \\
\hline $6 \sec A$ & $30 \mathrm{sec} B$ & $30 \mathrm{sec}$ & $\mathrm{A}$ B \\
\hline $6 \mathrm{sec} A$ & $6 \mathrm{sec} B$ & $30 \mathrm{sec}$ & $\mathrm{A}$ 早 \\
\hline
\end{tabular}

Figure 2. The experimental conditions used in Experiment 3. Only a single pair of objects appeared within a day.

rewarded, a delay interval of either 6 or $30 \mathrm{sec}$ ensued, followed by the choice test in which the sample was correct and rewarded.

The conditions listed in the bottom part of Figure 2 were designed to assess ISI effects. In these trial sequences, the ITI terminated with the incorrect choice stimulus being presented as the sample, followed by a variable ISI leading to the presentation of the correct choice stimulus as the sample, followed by a 30-sec delay interval and the choice test. The first two conditions exchanged ITI and ISI values so that the total time interval between the current choice test and the choice test on the preceding trial was held constant (at approximately $69 \mathrm{sec}$ ) while ISI varied. The final condition used a 6-sec ISI and ITI and thus was associated with a test-test interval of approximately $45 \mathrm{sec}$.

Counting the two delay intervals as different trial types, there were seven distinct trial sequences. Each of these seven sequences was presented four times each day for 24 days. In all, 29 trials were given each day, since a "dummy" trial (consisting of a random choice of one of the trial types ignoring ITI) was used as the first trial each day to set up the appropriate ITI conditions.

Within each day, only two different stimuli were used. Each of these two objects was correct on half of the 28 meaningful trials, and the position of the correct choice stimulus was randomized, balanced within a day, and subject to the further constraint that the correct object could occupy the same position on no more than four consecutive trials. In addition, consecutive trial relationships were balanced such that the likelihood that the same object was correct vs. incorrect and the likelihood that it occupied the same vs. the alternative position were equal. A different pair of junk stimuli was used for each monkey, and each day a new set of junk stimuli was introduced.

\section{Results}

As Table 3 indicates, overall performance was poor. The first four conditions in the table reveal both delay and ITI effects, with ITI effects increasing with delay interval. The next two conditions provide a direct test for ISI effects with ITI varied but intertest interval held constant. With total time between consecutive test trials equated, ISI had no effect. The condition with an ITI of $6 \mathrm{sec}$ and an ISI of $6 \mathrm{sec}$ did 
Table 3

Proportion Correct (PC) on the Various Combinations of Intertrial Intervals (ITIs), Intersample Intervals (ISIs), and Delays Used in Experiment 3

\begin{tabular}{rrrl} 
ITI & ISI & Delay & PC \\
\hline 6 & & 6 & .70 \\
6 & & 30 & .60 \\
30 & & 6 & .73 \\
30 & 6 & 30 & .69 \\
30 & 30 & 30 & .61 \\
6 & 6 & 30 & .62 \\
6 & 30 & .59 \\
\hline
\end{tabular}

Note-Each point is based on 400 observations.

not produce substantially worse performance than was observed in the other two conditions, in which both stimuli appeared as samples.

Analyses of variance confirmed these trends. One analysis examined ITI and delay effects, excluding the ISI conditions associated with within-trial proactive interference. The effects of ITI $[F(1,5)=31.83$, $\mathrm{MSe}=4.92, \mathrm{p}<.01]$, Delays $[\mathrm{F}(1,4)=9.65, \mathrm{MSe}=$ $25.38, \mathrm{p}<.05]$, and the interaction of ITI and Delays $[\mathrm{F}(1,4)=38.64, \mathrm{MSe}=1.32, \mathrm{p}<.01]$ all proved to be significant. A separate analysis of the within-trial proactive interference conditions failed to reveal any differences among these three trial types $(F<1)$, although the worst performance was associated with the condition having the smallest intertest interval.

\section{Discussion}

Once again, ISI effects failed to appear. Instead, lengthening the intertest interval proved to be the key factor in facilitating performance. Since the Jarvik et al. (1969) experiment did not separate the contributions of ISI from intertest interval, it is conceivable that their results similarly reflect intertest interval rather than ISI influences. Intertest interval effects were clear and were significant on single-sample trials; they were evident, but not significant, on trials using two samples. Since performance was so low on twosample trials, it is hard to make more direct comparisons with the Jarvik et al. (1969) results. Again, the absence of ISI effects creates problems for the temporal discrimination and trace strength competition theories, difficulties which it seems could be circumvented by modifying the theories to acknowledge the separate contributions of long-term memory and short-term processing of event information.

\section{GENERAL DISCUSSION}

In general, the experiments clearly demonstrated that the interval between an interfering stimulus and the to-be-remembered stimulus did not have effects commensurate with the delay involved. Although control trials provided an independent index that substantial forgetting occurred over the range of ISIs used, intersample interval did not affect performance in the first experiment, yielded nonmonotonic effects in the second, and once again produced no effect in a third experiment. In one sense, these are a set of null results in that no distinctive ISI effects were noted, but in another sense, the results are definitive in that the theories are inconsistent with the absence of ISI effects combined with the clear presence of delay effects. In their present form, neither the temporal discrimination theory nor the trace strength competition theory is able to reconcile these two main findings, because they imply an intimate link between ISI and delay effects.

The most straightforward way to modify the above theories is to give up the assumption of trace independence. One way to do this relies on the distinction between short- and long-term memory. Specifically, one could assume that, when a stimulus is presented, some long-term trace strength or temporal information builds up and at the same time a representation enters short-term memory. At the time of the test, performance can be based either on the longterm representation, if it can be accessed, or on shortterm memory, if the appropriate information still resides there. A key additional assumption is that if a second stimulus presentation occurs prior to a test, the representation of the first presentation tends to be displaced from short-term memory. In the standard interference conditions, stimulus B will displace stimulus A from short-term memory and choices of stimulus A would be based solely on its long-term strength. With this added assumption, a trace strength theory would be consistent with the absence of interpresentation interval effects and could account for why animals would choose the more recently presented stimulus of two stimuli that should be equal in strength (Roberts \& Grant, 1974). The more recent stimulus would always have the advantage of being represented in, and displacing other representations from, short-term memory. Corresponding assumptions could be added to a temporal discrimination theory to bring it more into line with the present results.

In many respects, the above modifications are a bit glib. In the introduction, it was argued that a combination of loss of information from long-term memory and gain of information from additional rehearsal time leads to the expectation that ISI and delay effects would not be comparable. But it is apparent that judicious use of two potentially offsetting factors can lead to expectations consistent with almost any result. Since many possible explanations of the data depend on conjectures about the strength and availability of the initially presented stimulus, tests involving the initial stimulus pitted against a 
new stimulus might be useful in providing an independent index of the strength of the initially presented sample.

General notions of short-term memory, by themselves, do not provide an explanation for either the apparent nonmonotone ISI effect noted in Experiment 2 or for the clear intertest interval effects observed in Experiment 3. To avoid piling speculation on speculation, it may be wise to let the theoretical import of the nonmonotone ISI effects await replication and clarification. For example, the ISI effects seem to be confined to the "different" trials, yet the ISI by the Same vs. Different interaction fell short of statistical significance.

It would doubtless be brash to claim that the present studies are completely reconciled with earlier evidence for ISI effects. Previous demonstrations of ISI effects all involve ISIs shorter than those used in the present studies, and perhaps ISI effects are not closely tied to delay intervals in the way that several prominent memory theories have implied.

The set of experiments, as a whole, is consistent in suggesting that variations on and alternatives to simple temporal discrimination and trace strength competition models will be needed in order for us to develop a more accurate account of proactive interference in animal memory. In particular, the observed pattern of ISI and delay effects suggests adding the assumption that information from both short- and long-term memory influences DMTS performance and that interactions among processes in short-term memory give rise to nonindependence of stimulus events. Experiments varying the similarity of alternative sample stimuli reinforce this conclusion (Medin et al., 1980).

\section{REFERENCES}

D'AмAто, M. R. Delayed matching and short-term memory in monkeys. In G. H. Bower (Ed.), The psychology of learning and motivation (Vol. 7). New York: Academic Press, 1973.

Grant, D. S., \& Roberts, W. A. Trace interaction in pigeon short-term memory. Journal of Experimental Psychology, 1973, 101, 21-29.

Herman, C. M. Interference and auditory short-term memory in the bottle nose dolphin. Animal Learning \& Behavior, 1975, $3,43-48$.

Herzog, H. L., Grand, D. S., \& Roberts, W. A. Effects of sample duration and spaced repetition upon delayed matchingto-sample in monkeys (Macaca arctoides and Saimiri sciureus). Animal Learning \& Behavior, 1977, 5, 347.354.

Jarvik, M. E., Goldfarb, T. L., \& Carley, J. L. Influence of interference on delayed matching in monkeys. Journal of Experimental Psychology, 1969, 81, 1-6.

Maki, W. S., Moe, J. C., \& Bierley, C. M. Short-term memory for stimuli, responses, and reinforcers. Journal of Experimental Psychology: Animal Behavior Processes, 1977, 3, 156-177.
Mason, M., \& Wilson, M. Temporal differentiation and recognition memory for visual stimuli in rhesus monkeys. Journal of Experimental Psychology, 1974, 103, 383-390.

MEDin, D. L. The role of reinforcement in discrimination learning set in monkeys. Psychological Bulletin, 1972, 77, 305-318.

MEDIN, D. L. The comparative study of memory. Journal of Human Evolution, 1974, 3, 455-463.

Medin, D. L., Reynolds, T. J., \& Parkinson, J. K. Stimulus similarity and retroactive interference and facilitation in monkey short-term memory. Journal of Experimental Psychology: Animal Behavior Processes, 1980, 6, 112-125.

Mishkin, M., \& Delacour, J. An analysis of short-term visual memory in the monkey. Journal of Experimental Psychology: Animal Behavior Processes, 1975, 1, 326-334.

REynolds, T. J., \& Medin, D. L. Strength vs. temporal-order information in delayed-matching-to-sample performance by monkeys. Animal Learning \& Behavior, 1979, 7, 294-300.

Roberts, W. A., \& Grant, D. S. Short-term memory in the pigeon with presentation time precisely controlled. Learning \& Motivation, 1974, 5, 393-408.

Roberts, W. A., \& Grant, D. S. Studies of short-term memory in the pigeon using the delayed matching-to-sample procedure. In D. L. Medin, W. A. Roberts, \& R. T. Davis (Eds.), Processes of animal memory. Hillsdale, N.J: Erlbaum, 1976.

SPEAR, N. E. Retrieval of memory in animals. Psychological Review, 1973, 80, 163-194.

Spear, N. E. Retrieval of memories. In W. K. Estes (Ed.), Handbook of learning and cognitive processes (Vol. 4). Hillsdale, N.J: Erlbaum, 1976.

Wagner, A. R., Rudy, J. W., \& Whitlow, J. W. Rehearsal in animal conditioning. Journal of Experimental Psychology, $1973,97,407-426$.

WILSON, M. Identification, discrimination, and retention of visual stimuli. In A. M. Schrier \& F. Stollnitz (Eds.), Behavior of nonhuman primates (Vol. 5). New York: Academic Press, 1974.

Worsham, R. W. Temporal discrimination factors in the delayed matching-to-sample task in monkeys. Animal Learning \& Behavior, 1975, 3, 93-97.

Zentall, T. R. Effect of context change on forgetting in rats. Journal of Experimental Psychology, 1970, 86, 440-448.

Zentall, T. R., \& Hogan, D. E. Memory in the pigeon: Proactive inhibition in a delayed matching task. Bulletin of the Psychonomic Society, 1974, 4, 109-112.

Zentall, T. R., \& HogAN, D. E. Short-term proactive inhibition in the pigeon. Learning Motivation, 1977, 8, 367-386.

\section{NOTE}

1. Nothing in this account necessarily requires long-term memory to be permanent. The main idea is simply that long-term memory should be persistent relative to short-term memory. In DMTS paradigms in which only a small number of alternative sample stimuli are used, there ought to be substantial between-trial proactive interference because the incorrect stimulus on a given trial might have been the correct stimulus on the just preceding trial. In those circumstances, it might be difficult to demonstrate very efficient performance at long delay intervals (one standard by which long-term memory is measured), not because there is no long-term memory for the current trial, but rather because there is, in addition, considerable long-term memory for earlier trials, which acts as a source of interference.

(Received for publication July 23, 1979; revision accepted May 12, 1980.) 